\title{
The radial augmentation index in children with Kawasaki disease without acute coronary artery lesions during the convalescent period
}

This article was published in the following Dove Press journal:

Therapeutics and Clinical Risk Management

\author{
Jianbo Zheng ${ }^{1,2}$ \\ Tsuneyuki Nakamura' \\ $\mathrm{Na} \mathrm{Lu}{ }^{1,2}$ \\ Kaori Hori' \\ Masato Oguri' \\ Masaru Sakurai ${ }^{3}$ \\ Masao Ishizaki ${ }^{3}$ \\ 'Department of Pediatric Cardiology, \\ Kanazawa Medical University, Ishikawa, \\ Japan; ${ }^{2}$ Department of Pediatrics, Union \\ Hospital, Tongii Medical College, \\ Huazhong University of Science and \\ Technology, Wuhan, People's Republic of \\ China; ${ }^{3}$ Department of Hygiene, \\ Kanazawa Medical University, Ishikawa, \\ Japan
}

Purpose: We recently reported that children and adolescents with a history of Kawasaki disease (KD) had slight but significant elastic arterial stiffness even when no coronary artery lesions (CALs) were present. Moreover, we hypothesized that KD-related arteriopathy may also cause peripheral artery dysfunction. The objective of this study was to assess the involvement of radial artery pulse waves, especially reflection waves from the peripheral arteries using the radial artery augmentation index (rAI) in patients without CALs after KD. Materials and methods: We first collected the rAI data from 312 subjects (149 consecutive patients of KD and 163 control subjects). Next, 225 cases between 6 and 15 years old were selected. Finally, 41 pairs were included for analysis. The rAI values of these two groups were compared and analyzed. Acute-phase data were also collected to reveal the possible correlation with rAI in the convalescent period.

Results: Multivariable analysis revealed the history of KD was positively correlated with rAI@75 value in children from 6 to 15 years old. After pairing the height and gender, the KD group also had significantly higher rAI and rAI@75 than the control group (rAI 60.63 \pm 13.77 vs $54.56 \pm 13.17, p=0.028$; rAI@75 63.61 \pm 15.21 vs $55.68 \pm 14.86, p=0.003$ ). With regard to acute-phase condition, nonresponse to initial treatment was also linked to elevated rAI during the convalescent period.

Conclusions: During the convalescent period, the rAI increased in KD patients without acute CALs. Furthermore, nonresponse to initial treatment in acute phase conferred higher rAI to KD subjects than respondent cases. Elevated rAI means the reflection wave from the peripheral vascular is stronger or earlier. This small but significant change may indicate the existence of peripheral artery stiffness during the convalescent period.

Keywords: Kawasaki disease, augmentation index, peripheral artery stiffness

\section{Background}

Kawasaki disease (KD) is an acute, self-limited vasculitis of unknown etiology that occurs predominantly in infants and young children. ${ }^{1}$ It is characterized by fever, bilateral nonexudative conjunctivitis, erythema of the lips and oral mucosa, extremity changes, rash, and cervical lymphadenopathy. The systemic vasculitis mainly affects medium-sized arteries in multiple systems, especially the coronary arteries. Administration of high-dose intravenous immunoglobulin reduces the incidence of coronary artery lesions (CALs) from $20 \%$ to $25 \%$ to $<5 \%$ in the acute phase. ${ }^{1}$

As a systemic vasculitis, KD damages not only the medium-sized arteries but also the small arteries and veins. ${ }^{2}$ Although $\mathrm{KD}$ is self-limited, there are pathological
Correspondence: Tsuneyuki Nakamura Department of Pediatric Cardiology, Kanazawa Medical University, I Chome-I Daigaku, Uchinada, Kahoku District, Ishikawa, Japan

Email306919303@qq.com 
changes in the convalescent period, including infiltration of the vascular wall by macrophages, lymphocytes (primarily CD8+ T cells), and plasma cells, ${ }^{3}$ whichcan cause artery wall remodeling. These changes may cause systemic artery stiffness and damage vascular function in the late phase of $\mathrm{KD}$ and thus infer a higher risk of cardiovascular disease. Some studies have reported that KD can cause both structural and functional systemic abnormalities that persist long after the acute phase. $^{4-7}$ Other studies have verified the association between $\mathrm{KD}$ and atherosclerosis. ${ }^{8-12}$

We recently reported that children and adolescents with a history of KD have slight but significant elastic arterial stiffness despite having no CALs. ${ }^{13}$ We hypothesized that KD-related arteriopathy also affected peripheral artery function other than central artery function. As a parameter associated with wave reflection and the stiffness of resistance vessels, the radial artery augmentation index (rAI) can reflect systemic artery stiffness,especially in the peripheral sites. Our study collected the rAI data in both patients with $\mathrm{KD}$ and healthy controls in order to investigate possible differences between the two groups.

\section{Materials and methods}

\section{Study subjects}

We conducted the study from May 2013 to April 2016 in the pediatric department of a hospital affiliated with the Kanazawa Medical University. Consecutive pediatric patients older than 4 years with a diagnosis of $\mathrm{KD}$ for $>1$ month were recruited. We used the Japanese criteria (the fifth revised edition in 2002) for the diagnosis of KD. All patients were given standard treatment, including intravenous immunoglobulin and high doses of aspirin (30-50 mg/kg). Unaffected subjects mainly in the same age range without a history of $\mathrm{KD}$ were assigned as control group. Some of them were the children whose parents worked in our hospital and the others were the ones who took a health check in our department. All subjects in both groups were Japanese nonsmokers without a medical history of hypertension, hyperglycemia,or hypercholesterolemia. None had a known family history of hereditary disease. This study was approved by the ethics committee of Kanazawa Medical University (the approval number: NO. R213), and written informed consent for participation was obtained from all subjects or from their parents.

\section{Study protocol}

We reviewed the medical history of all participants and measured their body weight (BW), height (HT), heart rate
(HR), and blood pressure (BP). Body mass index (BMI) was calculated using the formula $\mathrm{BW} / \mathrm{H}^{2}$. The acute-phase clinical data of patients with KD were reviewed carefully to collect the following details: age at disease onset, time interval between disease onset and the date of the examination for the study, and whether CALs were present or not. We used the Japanese criteria (fifth revised edition) to identify CALs, which were considered present if any of the following criteria were met: 1) the internal lumen diameter was $>3 \mathrm{~mm}$ in children under 5 years or $>4 \mathrm{~mm}$ in children 5 years or older; 2) the internal diameter of any segment was at least 1.5 -fold larger than that of an adjacent segment; or 3 ) the coronary artery lumen was conspicuously irregular. Subjects with CALs during the acute phase were excluded from our study. rAI measurement is described below.

\section{rAl measurements}

The Omron HEM-9000AI (Omron Healthcare Co., Ltd, Japan) was used to measure the rAI. Participants were advised not to consume food or drink except for water for at least $3 \mathrm{hrs}$ before the rAI examination. After resting for at least 15 mins in a quiet examination room, each subject was examined in a sitting position by experienced doctors. Due to the necessary cooperation problem, we only included children who were over 4 years old. For the younger children, in addition to the operator, a nurse and the caregiver (mother) were present in the room throughout the assessment. The nurse played an ageappropriate video to the child before and during the procedure. The assessment did not start until the child was comfortable and the questions of the child and caregiver were answered. After the preparation, the pulse wave sensor unit was placed over the radial artery pulse spot and fixed. The radial artery waveform (Figure 1) was recorded, and the rAI was calculated using the formula $\mathrm{rAI}=(\mathrm{P} 2 / \mathrm{P} 1 * 100)$, where systolic peak pressure $2(\mathrm{P} 2)$ is the late systolic pressure and systolic peak pressure 1 (P1) is the early systolic pressure. As the rAI may be influenced by heart rate, it was adjusted to 75 bpm (rAI@75) for heart rate standardization.

\section{Data including procedure}

Primarily, 312 subjects were recruited. The children younger than 6 years old were excluded because of the cooperation problem during the AI measurement even after full preparation. According to Tomoaki Murakami's $^{14}$ and EV Hidvégi's study, ${ }^{15}$ the linear 


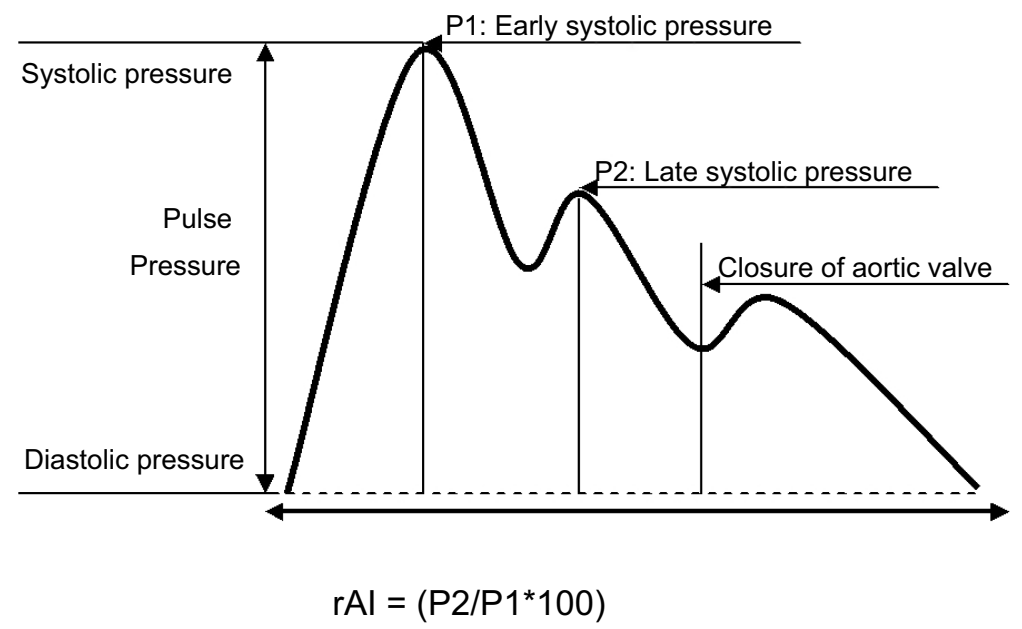

Figure I Radial artery waveform.

Abbreviations: $\mathrm{rAl}$, radial artery augmentation index; P2, systolic peak pressure 2; PI, systolic peak pressure I.

decreasing trend of AI changed around 15 years old; we thus have not included the cases over 15 years old for more accurate data analysis. Moreover, 5 cases reached the criteria of hypertention ${ }^{16}$ and 25 cases had an abnormal BMI index ( $\geq 2 \mathrm{SD})$ according to WHO child growth standards 2006; they were also excluded. Finally, 225 cases were included and 41 pairs were matched by the same height and gender considering these two factors in determing AI value in healthy children. ${ }^{14,15}$ The whole procedure was summarized in Figure 2

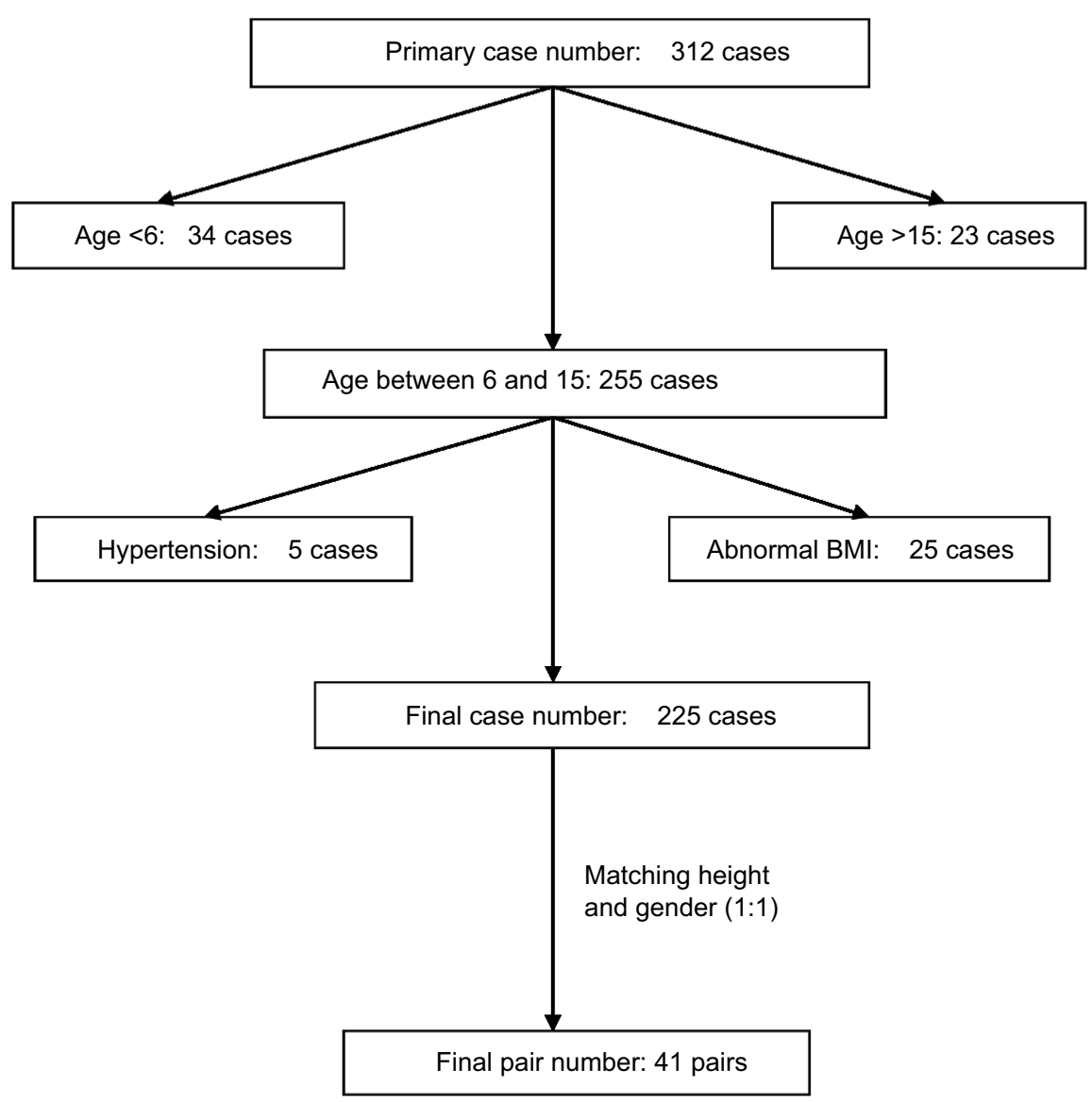

Figure 2 Data including procedure. 


\section{Statistical analysis}

Continuous data were expressed as mean \pm SD and nonparametric statistical analysis was used. Categorical data were analyzed using the Chi-square test. We performed multivariable regression analysis using the rAI@75 as the dependent variable. Paired-samples $T$ test was employed for paired data analysis. Statistical analyses were performed using the SPSS statistical software package, version 16.0. Missing values were excluded from the analysis. A two-sided $p$-value $<0.05$ was considered to indicate statistical significance.

\section{Results}

\section{Clinical characteristics}

The characteristics of the 225 subjects (123 healthy controls and $102 \mathrm{KD}$ subjects) are listed in Table 1. There was no significant difference in the proportion of boys and girls between KD group and control group $(p=0.539)$. The KD group was a little younger than the control group, but this difference was not statistically significant. The KD group had significantly lower values in terms of height and BW $(p<0.01)$. With regard to $\mathrm{BP}$, the diastolic pressure is a little higher in the $\mathrm{KD}$ group than the control group ( $p=0.048$ ), whereas no difference existed in systolic pressure and pulse pressure. Although the rAI and rAI@75 of KD group were significantly higher than the healthy control group, further analysis was needed due to the difference in height and other factors between these two groups.

\section{KD history was positively correlated with rAI and rAI@75}

Because of the significant difference in height and other factors between KD and healthy controls, univariable

Table I Subject characteristics

\begin{tabular}{|c|c|c|c|}
\hline & $\begin{array}{l}\text { Control } \\
\text { (mean } \pm \text { SD) }\end{array}$ & $\begin{array}{l}\text { KD } \\
(\text { mean } \pm \text { SD) }\end{array}$ & $p$-value \\
\hline Age (years) & $11.22 \pm 2.73$ & $10.54 \pm 2.66$ & $0.060 *$ \\
\hline Height $(\mathrm{cm})$ & $|45.27 \pm| 7.42$ & $138.72 \pm 17.19$ & $0.005 *$ \\
\hline Male proportion (\%) & $71 / 123(57.7 \%)$ & $63 / 102$ (61.8\%) & $0.539^{\dagger}$ \\
\hline Body weight (kg) & $38.14 \pm 11.83$ & $33.69 \pm 11.45$ & $0.009 *$ \\
\hline $\begin{array}{l}\text { Systolic pressure } \\
(\mathrm{mmHg})\end{array}$ & $103.12 \pm 8.18$ & $103.11 \pm 8.89$ & $0.884 *$ \\
\hline $\begin{array}{l}\text { Diastolic pressure } \\
(\mathrm{mmHg})\end{array}$ & $60.60 \pm 8.47$ & $62.63 \pm 6.49$ & $0.048 *$ \\
\hline Pulse pressure $(\mathrm{mmHg})$ & $42.52 \pm 7.63$ & $40.48 \pm 7.92$ & $0.059 *$ \\
\hline rAl & $56.63 \pm 14.44$ & $62.5 I \pm|4.4|$ & $0.003 *$ \\
\hline rAI@75 & $57.60 \pm 16.15$ & $66.00 \pm 15.92$ & $<0.001 *$ \\
\hline
\end{tabular}

Notes: ${ }^{\dagger}$ Pearson Chi-square test was used. *Mann-Whitney $U$ test was used. Abbreviations: KD, Kawasaki disease; rAl, radial artery augmentation index; rAI@75, radial artery augmentation index adjusted to heart rate in 75 bpm. analysis to compare the rAI between $\mathrm{KD}$ and healthy control group makes little sense. We conducted multivariable regression analysis to reveal the correlation between KD history and rAI. Tables 2 and 3 show height was strongly correlated with rAI and rAI@75. The elevated height always links with decreased rAI and rAI@75. The same negative correlation was also found between male sex and rAI@75, with females having a higher rAI than males. In contrast, $\mathrm{KD}$ history was positively correlated with rAI and rAI@75. In other words, the subjects with KD history had higher rAI@75 than healthy controls. Due to the strong correlation between height and rAI, we adopted these two variables to draw a scatter plot to better illustrate the relationship between KD history and rAI. Figures 3 and 4 also indicate the positive correlation between KD history and rAI and rAI@75.

\section{Elevated rAl and rAI@75 in the KD group after matching height and gender}

According to our results of multivariable analysis and linear correlation of scatter plot, height had a strong negative correlation with rAI and rAI@75; female sex also correlated with elevated rAI and rAI@75. We paired the KD subjects and healthy controls by matching height and sex (ratio 1:1). 41 pairs were finally included with the same height and sex in each pair. Table 4 shows that there was also no significant difference between KD group and healthy controls in terms of age, body surface area (BSA), and BMI. Paired-Samples $t$-test shows that KD group had significantly higher rAI and rAI@75 than the control group (rAI $60.63 \pm 13.77$ vs $54.56 \pm 13.17, p=0.028$; rAI@75 63.61 \pm 15.21 vs 55.68 $\pm 14.86, p=0.003)$. This difference is illustrated in Figure 5. With regard to heart rate, systolic pressure, and diastolic pressure, no significant difference was found between these two groups.

\section{Initial intravenous immunoglobulin (IVIG) nonresponse was positively correlated with rAl, whereas fever duration had negative correlation}

In order to reveal the relationship between acute-phase condition and convalescent rAI, we also performed multivariable linear regression analysis. Table 5 shows that initial IVIG nonresponse was positively correlated with rAI. In contrast, fever duration was negatively correlated with rAI and rAI@75. These results mean that the history of nonresponse to initial IVIG in acute phase would further increase $\mathrm{rAI}$ in KD patients, whereas longer fever duration has the opposite effect. But after excluding the 
Table 2 Multivariable linear regression analysis for $\mathrm{rAl}$

\begin{tabular}{|l|l|l|}
\hline & $\begin{array}{l}\text { Standard } \\
\text { coefficient }\end{array}$ & -value \\
\hline Age (per year) & 0.009 & 0.945 \\
Body weight (per kg) & 0.156 & 0.382 \\
Sex (male vs female) & -0.088 & 0.093 \\
Height (per cm) & -0.763 & $<0.001 * * *$ \\
KD history (KD vs control) & 0.092 & 0.086 \\
\hline
\end{tabular}

Note: $* * * p<0.001$.

Abbreviations: KD, Kawasaki disease; rAl, radial artery augmentation index.

Table 3 Multivariable linear regression analysis for rAI@75

\begin{tabular}{|l|l|l|}
\hline & $\begin{array}{l}\text { Standard } \\
\text { coefficient }\end{array}$ & $p$-value \\
\hline Age (per year) & -0.118 & 0.305 \\
Body weight (per kg) & 0.072 & 0.635 \\
Sex (male vs female) & -0.115 & $0.010^{*}$ \\
Height (per cm) & -0.664 & $<0.001 * * *$ \\
KD history (KD vs & 0.133 & $0.004^{* *}$ \\
control) & & \\
\hline
\end{tabular}

Notes: $* p<0.05, * * p<0.01, * * * p<0.001$.

Abbreviations: $\mathrm{KD}$, Kawasaki disease; rAl, radial artery augmentation index; rAI@75, radial artery augmentation index adjusted to heart rate in 75 bpm. cases $(\mathrm{n}=9$ ) whose fever duration was $<5$ days, no correlation was found between fever duration and $\mathrm{rAI}(\beta=-0.081$, $p=0.419)$ anymore. With respect to white blood cell (WBC) and C-reactive protein (CRP) in acute phase, we found no correlation with rAI or rAI@75 in the convalescent period.

\section{Discussion}

$\mathrm{KD}$ is thought to be an acute and self-limited vasculitis. Most researches focus on changes that occur during the acute phase,especially changes in the medium-sized coronary artery. In the convalescent period, long-term followup is suggested for patients with persistent CALs, but sequels are not frequently studied in patients without CALs during the acute phase. Due to challenges in animal models and a lack of pathological samples, it is difficult to perform a direct and comprehensive evaluation of the vascular system. Indirect and noninvasive surrogate measures that reflect vascular function are thus important in research. As an index of vascular function, $r$ AI can reflect artery stiffness, especially stiffness in the peripheral artery.

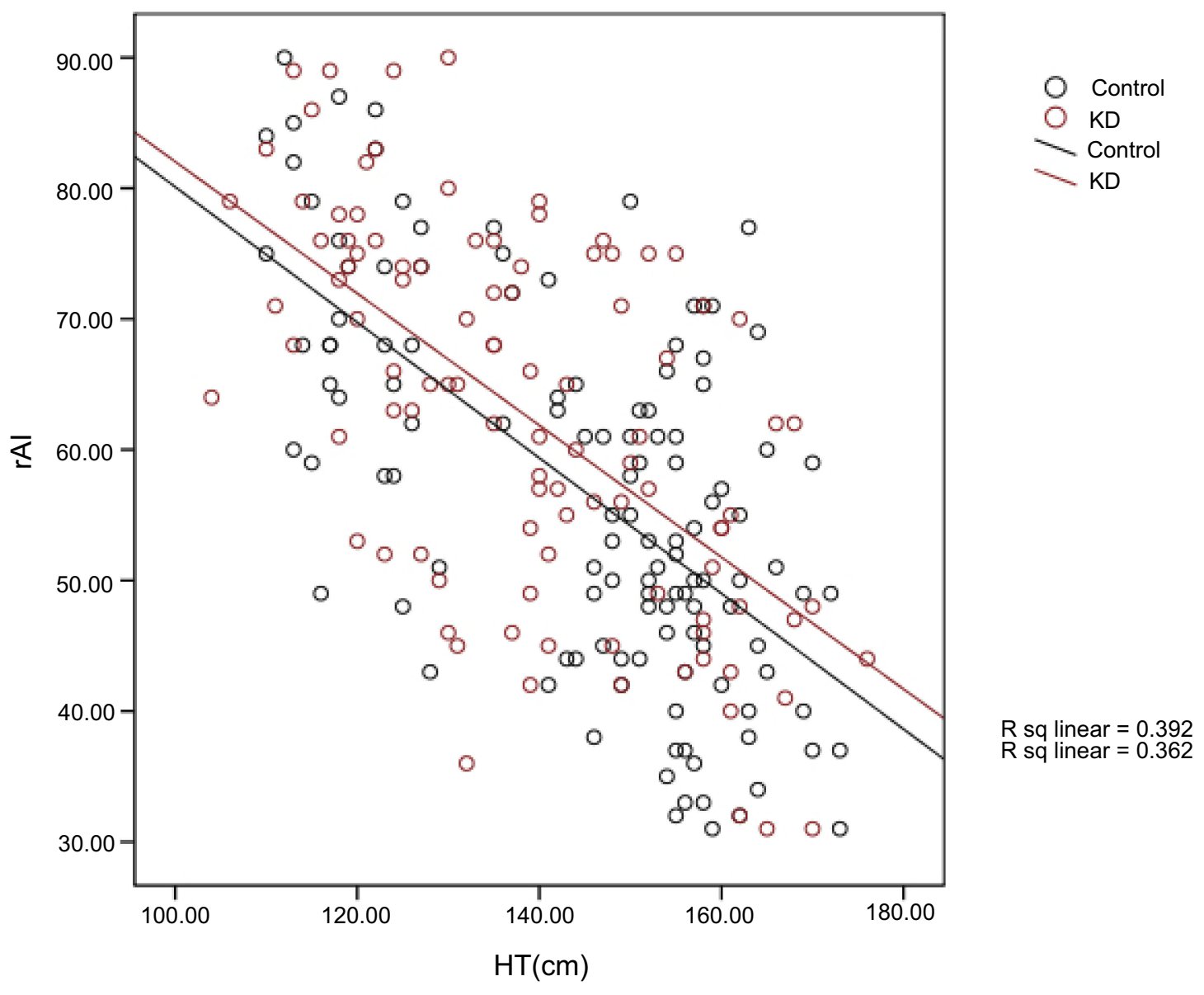

Figure 3 Linear correlation between $\mathrm{rAl}$ and height. 


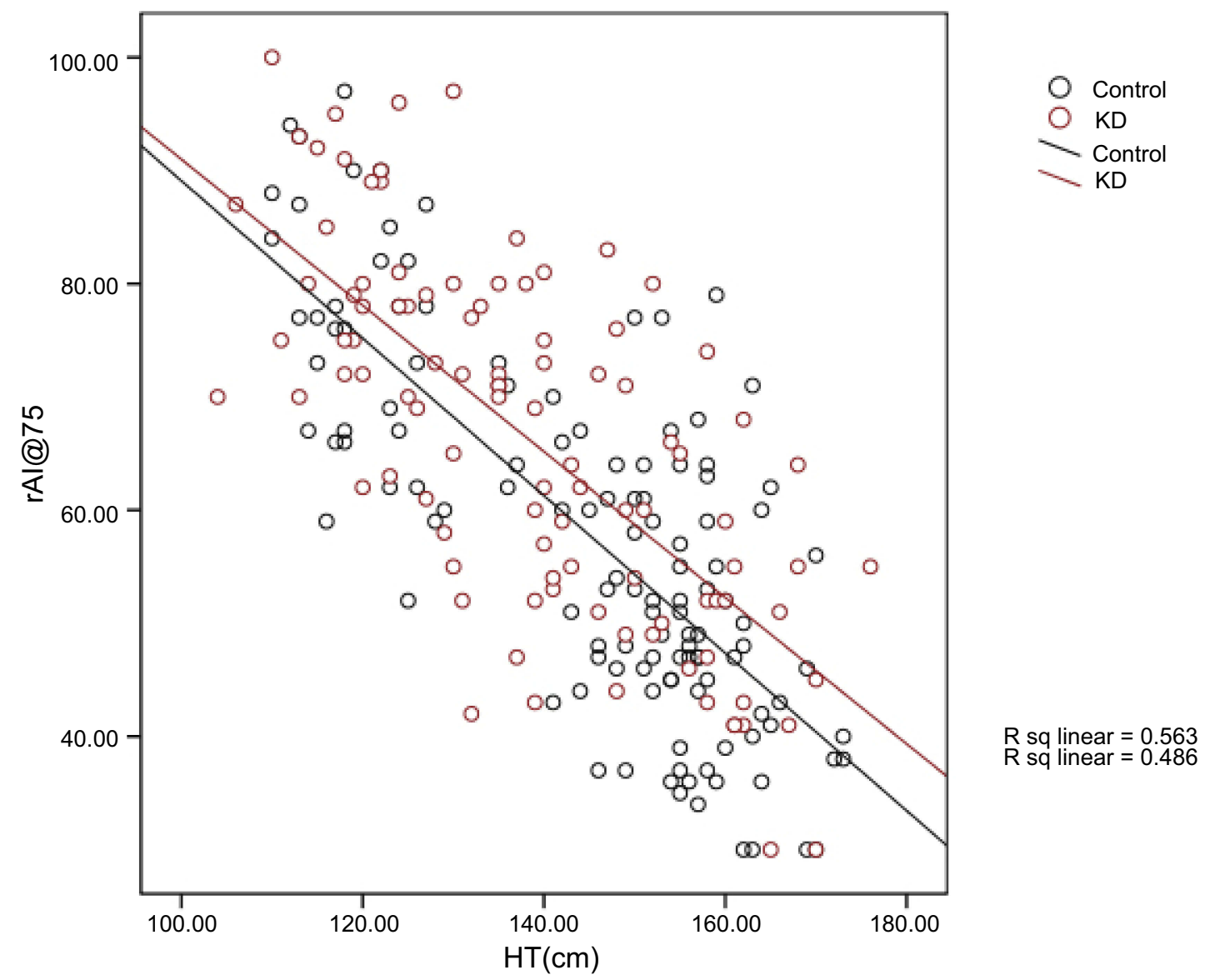

Figure 4 Linear correlation between rAl@75 and height.

Abbreviations: KD, Kawasaki disease; rAI, radial artery augmentation index; rAI@75, radial artery augmentation index adjusted to heart rate in 75 bpm.

Table 4 Paired $t$-test after matching height and sex (I:I)

\begin{tabular}{|c|c|c|c|}
\hline & $\begin{array}{l}\text { Control } \\
(\text { mean } \pm \text { SD) }\end{array}$ & $\begin{array}{l}\text { KD } \\
\text { (mean } \pm \text { SD) }\end{array}$ & $p$-value \\
\hline Age (years) & $10.86 \pm 2.73$ & $11.25 \pm 2.59$ & 0.151 \\
\hline $\operatorname{BSA}\left(\mathrm{m}^{2}\right)$ & $1.20 \pm 0.25$ & $1.19 \pm 0.24$ & 0.352 \\
\hline BMI $\left(\mathrm{kg} / \mathrm{m}^{2}\right)$ & $17.14 \pm 2.08$ & $17.09 \pm 1.99$ & 0.861 \\
\hline Heart rate (bpm) & $77.80 \pm 13.14$ & $81.37 \pm 13.63$ & 0.116 \\
\hline $\begin{array}{l}\text { Systolic pressure } \\
(\mathrm{mmHg})\end{array}$ & $101.54 \pm 6.82$ & $103.63 \pm 8.05$ & 0.103 \\
\hline $\begin{array}{l}\text { Diastolic pressure } \\
(\mathrm{mmHg})\end{array}$ & $59.78 \pm 7.03$ & $62.15 \pm 5.98$ & 0.059 \\
\hline $\begin{array}{l}\text { Pulse pressure } \\
(\mathrm{mmHg})\end{array}$ & $41.76 \pm 7.72$ & $41.49 \pm 8.23$ & 0.847 \\
\hline rAl & $54.56 \pm 13.17$ & $60.63 \pm 13.77$ & $0.028 *$ \\
\hline rAI@75 & $55.68 \pm 14.86$ & $63.61 \pm 15.21$ & $0.003 * *$ \\
\hline
\end{tabular}

Notes: $* p<0.05, * * p<0.01$

Abbreviations: KD, Kawasaki disease; rAl, radial artery augmentation index; rAI@75, radial artery augmentation index adjusted to heart rate in 75 bpm.

Until now, very few studies have investigated AI changes in $\mathrm{KD}$, and the results are controversial. Tobayama et al demonstrated that KD subjects had higher AI values than healthy controls, ${ }^{17}$ but Pinto FF found no difference. ${ }^{18}$ Both studies had small sample sizes, so larger samples are needed to settle this controversy.

Our study finally enrolled 225 subjects with an age from 6 to 15 years old, 102 patients with KD and 123 healthy controls, and the time interval from disease onset until the study examination ranged from 1 month to nearly 14 years. To the best of our knowledge, this is the only study of AI in a large sample of patients with KD. Our research showed that the rAI was a little but significantly higher in KD patients without CALs during the acute phase than in healthy controls. With regard to acute-phase condition, we found that initial IVIG nonresponse was positively correlated with rAI. This finding is consistent with the phenomenon that IVIG nonresponse was correlated with increased risk of CALs and thus enhanced vascular damage. One confusing finding in our study is that fever duration was negatively correlated with rAI andrAI@75. It seems contradictory with Mori's opinion $^{19}$ that persistent high fever for more than 10 days 


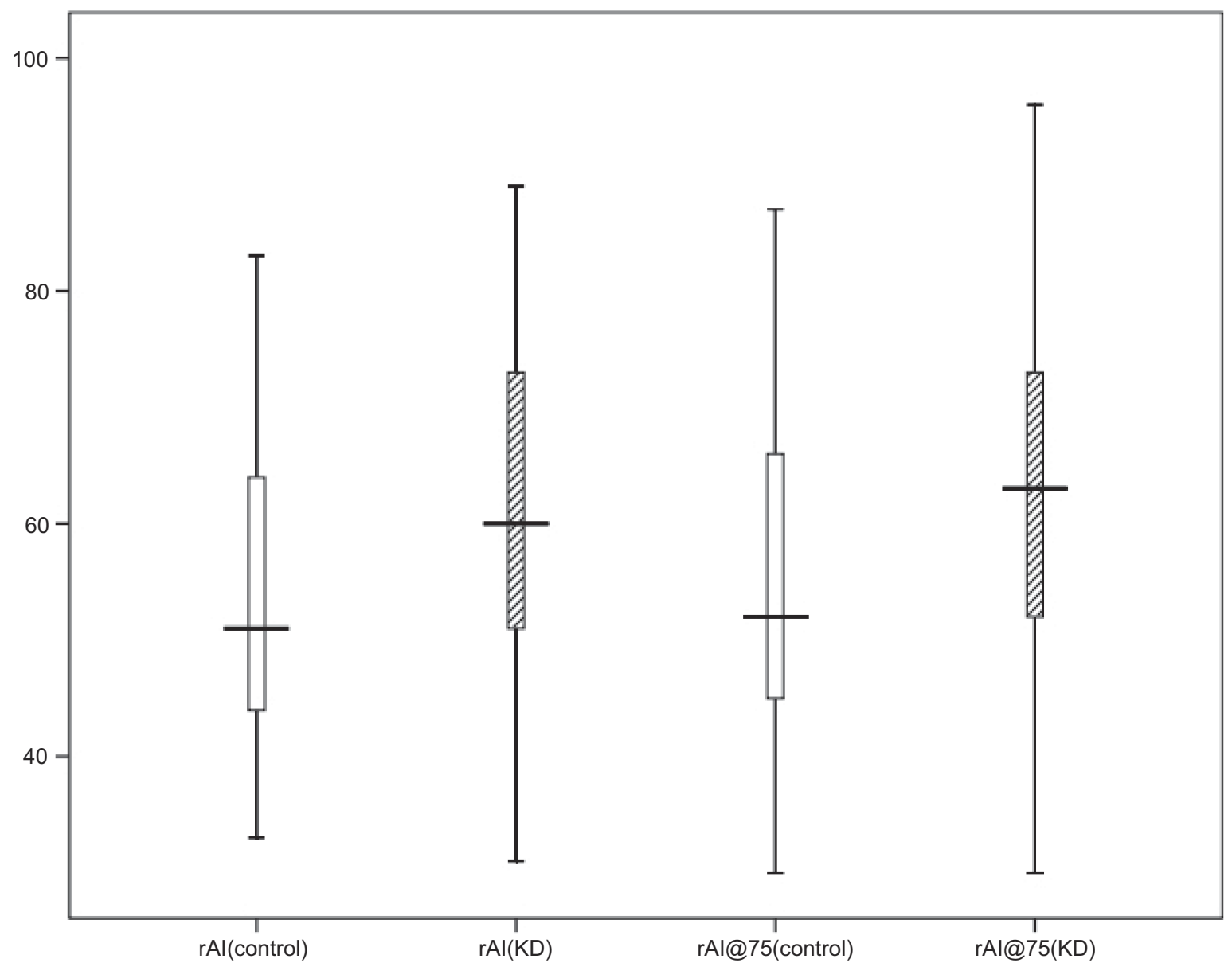

Figure 5 Comparison of rAl and rAI@75 after matching HT and sex.

Abbreviations: rAl, radial artery augmentation index; rAI@75, radial artery augmentation index adjusted to heart rate in 75 bpm.

Table 5 Multivariable regression analysis for rAI and rAI@75 in KD subjects

\begin{tabular}{|c|c|c|c|c|c|c|}
\hline & Mean士SD & Range & $\begin{array}{l}\beta \\
(r A I)\end{array}$ & $\begin{array}{l}\text { p-value } \\
\text { (rAI) }\end{array}$ & $\begin{array}{l}\beta \\
(r A I @ 75)\end{array}$ & $\begin{array}{l}\text { P-value } \\
\text { (rAl@75) }\end{array}$ \\
\hline Height $(\mathrm{cm})$ & $138.72 \pm 17.19$ & $104-176$ & -0.542 & $<0.001$ & -0.641 & $<0.001$ \\
\hline Sex (male proportion) & $63 / 102$ & $61.76 \%$ & -0.198 & $0.027 * *$ & -0.203 & $0.011 * *$ \\
\hline White blood cell $\left(10^{9} / \mathrm{L}\right)$ & $13.64 \pm 5.36$ & $4.92-35.42$ & -0.069 & 0.484 & -0.04 & 0.65 \\
\hline C-reactive protein $(\mathrm{mg} / \mathrm{L})$ & $7.36 \pm 6.02$ & $0.3-27.64$ & -0.011 & 0.919 & -0.008 & 0.936 \\
\hline Intravenous immunoglobulin (nonresponder proportion) & $21 / 102$ & $20.5 \%$ & 0.181 & $0.085 *$ & 0.112 & 0.232 \\
\hline Fever duration (days) & $6.55 \pm 1.82$ & $3-10$ & -0.198 & $0.056 *$ & -0.2 & $0.031 * *$ \\
\hline
\end{tabular}

Notes: $* p<0.1, * * p<0.05$

Abbreviations: KD, Kawasaki disease; rAl, radial artery augmentation index; rAI@75, radial artery augmentation index adjusted to heart rate in 75 bpm.

during the acute phase was related to endothelial dysfunction during the convalescent period. But in our study, the fever duration ranged from 3 to 10 days, and all subjects had fever resolution within 10 days. According to Egami $^{20}$ and Kobayashi's ${ }^{21}$ research, the one who received initial IVIG before the fifth day of disease onset tended to experience IVIG resistance, whereas no difference was found for the subjects who received the first dose of IVIG between the fifth and tenth day. We thus speculated that this certain fever duration distribution in our study may explain the negative correlation. Multivariable analysis after excuding KD cases who began initial treatment before day 5 after onset verified our speculation, but further studies are needed to confirm this result. The increased value of rAI in patients with KD may be due to peripheral vascular changes in the 
convalescent period. As the rAI is an index that is strongly related to the peripheral artery and vascular bed, this suggested that not only medium-sized arteries were affected by KD, but also smaller arteries. In adults, central aortic AI had been demonstrated as an independent predictor of mortality in end-stage renal disease patients. $^{22,23}$ Moreover, its predictive value of $\mathrm{CV}$ events in patients undergoing percutaneous coronary intervention and the hypertensive patients has also been verified; in children, although many pathological conditions such asobesity, diabetes, ${ }^{24}$ systemic hypertension, ${ }^{25}$ insulin resistance, $^{26}$ dyslipidemia, ${ }^{27}$ and chronic kidney disease $^{28}$ have been linked with increased AI, due to the lack of long-term follow-up studies, the clinical importance of AI in children is still unclear and needed to be clarified.

However, our study has some limitations. We have not detected the serum lipid profile in our subjects, although they did not have a dyslipidemia history. The difference in serum lipid and cholesterol between groups may contribute to rAI change. Second, our study was not longitudinal, and data collection was not taken at every time point. It is possible that periodic data collection is needed to better reflect dynamic changes in vascular function. Third, our study just analyzed the rAI difference in children between 6 and 15 years old; this conclusion cannot be applied to an extended age range. Last, our study only included Japanese patients, and the results may be different in other ethnic groups.

\section{Conclusions}

In conclusion, the rAI values were higher in KD patients without acute-phase CALs than in healthy controls. Elevated rAI means the reflection wave from the peripheral vascular is stronger or earlier. This small but significant change may indicate the existence of peripheral artery stiffness during the convalescent period in $\mathrm{KD}$ patients without acute CALs. It implied the peripheral vascular system was not in ideal health even when no acute CALs happened.

\section{Abbreviation list}

BW, body weight; HT, height; HR, heart rate; BP, blood pressure; BMI, body mass index; BSA, body surface area; $\mathrm{KD}$, Kawasaki disease; IVIG, intravenous immunoglobulin; WBC, white blood cell; CRP, C-reactive protein; CALs, coronary artery lesions; rAI, radial artery augmentation index; rAI@75, radial augmentation index adjusted to heart rate $75 \mathrm{bpm}$.

\section{Ethics approval and consent to participate}

The research has been performed in accordance with the Declaration of Helsinki and was approved by the ethics committee of Kanazawa Medical University (the approval number: NO.R213), and written informed consent for participation was obtained from all subjects or from their parents.

\section{Consent for publication}

There are no details on individuals reported within the manuscript; therefore, consent for publication is not required.

\section{Availability of data and materials}

The primary data are open access on figshare and the DOI is as follows:

https://doi.org/10.6084/m9.figshare.6689660

\section{Author contributions}

All authors contributed to data analysis, drafting or revising the article, gave final approval of the version to be published, and agree to be accountable for all aspects of the work.

\section{Disclosure}

The authors declare that they have no competing interests in this work.

\section{References}

1. Newburger JW. Diagnosis, treatment, and long-term management of Kawasaki disease: a statement for health professionals from the committee on rheumatic fever, endocarditis and Kawasaki disease, council on cardiovascular disease in the young, American heart association. Circulation. 2004;110(17):2747-2771. doi:10.1161/01. CIR.0000145143.19711.78

2. Shigeru Amano, Hazama F, Hamashima Y. Pathology of Kawasaki disease. Jpn Circ J. 1979;43(7):633-643.

3. Kliegman RM, Bonita S, St. Geme JW III, Behrman RE. Nelson Textbook of Pediatrics. 19th ed. Philadelphia, Pa, USA: Elsevier; 2011.

4. Senzaki H, Masutani S, Kobayashi J, et al. Circulating matrix metalloproteinases and their inhibitors in patients with Kawasaki disease. Circulation. 2001;104(8):860-863.

5. Senzaki H. The pathophysiology of coronary artery aneurysms in Kawasaki disease: role of matrix metalloproteinases. Arch Dis Child. 2006;91(10):847-851. doi:10.1136/adc.2005.087437

6. Takahashi K, Oharaseki T, Yokouchi Y, Hiruta N, Naoe S. Kawasaki disease as a systemic vasculitis in childhood. Ann Vasc Dis. 2010;3 (3):173-181. doi:10.3400/avd.sasvp01003 
7. Masuda H, Shozawa T, Naoe S, Tanaka N. The intercostal artery in Kawasaki disease. A pathologic study of 17 autopsy cases. Arch Pathol Lab Med. 1986;110(12):1136-1142.

8. Gupta-Malhotra M, Gruber D, Abraham SS, et al. Atherosclerosis in survivors of Kawasaki disease. J Pediatr. 2009;155(4):572-577. doi:10.1016/j.jpeds.2009.04.054

9. McCrindle BW, McIntyre S, Kim C, Lin T, Adeli K. Are patients after Kawasaki disease at increased risk for accelerated atherosclerosis? J Pediatr. 2007;151(3):244-248, 248 e241. doi:10.1016/j.jpeds.2007.03.056

10. Kadono T, Sugiyama H, Hoshiai M, et al. Endothelial function evaluated by flow-mediated dilatation in pediatric vascular disease. Pediatr Cardiol. 2005;26(4):385-390. doi:10.1007/s00246-004-0755-9

11. Newburger JW, Burns JC, Beiser AS, Loscalzo J. Altered lipid profile after Kawasaki syndrome. Circulation. 1991;84(2):625-631.

12. Fukunaga H, Kishiro M, Akimoto K, Ohtsuka Y, Nagata S, Shimizu T. Imbalance of peroxisome proliferator-activated receptor gamma and adiponectin predisposes Kawasaki disease patients to developing atherosclerosis. Pediatr Int. 2010;52(5):795-800. doi:10.1111/j.1442-200X.2010.03160.x

13. Oguri M, Nakamura T, Tamanuki K, et al. Subclinical arterial stiffness in young children after Kawasaki disease. Cardiol Young. 2014;24(1):87-94. doi:10.1017/S1047951112002302

14. Murakami T, Takeda A, Takei K, et al. Aortic pressure wave reflection in children. Hypertens Res. 2010;33(3):225-228. doi:10.1038/ hr.2009.218

15. Hidvegi EV, Illyes M, Molnar FT, Cziraki A. Influence of body height on aortic systolic pressure augmentation and wave reflection in childhood. J Hum Hypertens. 2015;29(8):495-501. doi:10.1038/ jhh.2014.118

16. Flynn JT, Kaelber DC, Baker-Smith CM, et al. Clinical practice guideline for screening and management of high blood pressure in children and adolescents. Pediatrics. 2017;140(3). doi:10.1542/ peds.2017-0685.

17. Tobayama H, Takahashi K, Fukunaga H, et al. Analysis of arterial function in adults with a history of Kawasaki disease. J Cardiol. 2013;61(5):330-335. doi:10.1016/j.jjcc.2012.12.007

18. Pinto FF, Laranjo S, Parames F, Freitas I, Mota-Carmo M. Long-term evaluation of endothelial function in Kawasaki disease patients. Cardiol Young. 2013;23(4):517-522. doi:10.1017/S1047951112001357
19. Mori Y, Katayama H, Kishi K, Ozaki N, Shimizu T, Tamai H. Persistent high fever for more than 10 days during acute phase is a risk factor for endothelial dysfunction in children with a history of Kawasaki disease. J Cardiol. 2016;68(1):71-75. doi:10.1016/j. jjcc.2015.08.008

20. Egami K, Muta H, Ishii M, et al. Prediction of resistance to intravenous immunoglobulin treatment in patients with Kawasaki disease. J Pediatr. 2006;149(2):237-240. doi:10.1016/j.jpeds.2006. 03.050

21. Kobayashi T, Inoue Y, Takeuchi K, et al. Prediction of intravenous immunoglobulin unresponsiveness in patients with Kawasaki disease. Circulation. 2006;113(22):2606-2612. doi:10.1161/CIRCULATI ONAHA.105.592865

22. London GM, Blacher J, Pannier B, Guerin AP, Marchais SJ, Safar ME. Arterial wave reflections and survival in end-stage renal failure. Hypertension. 2001;38(3):434-438.

23. Safar ME, Blacher J, Pannier B, et al. Central pulse pressure and mortality in end-stage renal disease. Hypertension. 2002;39 (3):735-738.

24. Urbina EM, Kimball TR, Khoury PR, Daniels SR, Dolan LM. Increased arterial stiffness is found in adolescents with obesity or obesity-related type 2 diabetes mellitus. J Hypertens. 2010;28 (8):1692-1698. doi:10.1097/HJH.0b013e32833a6132

25. Urbina EM, Khoury PR, McCoy C, Daniels SR, Kimball TR, Dolan LM. Cardiac and vascular consequences of pre-hypertension in youth. $J$ Clin Hypertens (Greenwich). 2011;13(5):332-342. doi:10.1111/j.1751-7176.2011.00471.x

26. Urbina EM, Gao Z, Khoury PR, Martin LJ, Dolan LM. Insulin resistance and arterial stiffness in healthy adolescents and young adults. Diabetologia. 2012;55(3):625-631. doi:10.1007/s00125-0112412-1

27. Urbina EM, Khoury PR, McCoy CE, Dolan LM, Daniels SR, Kimball TR. Triglyceride to HDL-C ratio and increased arterial stiffness in children, adolescents, and young adults. Pediatrics. 2013;131(4):e1082-e1090. doi:10.1542/peds.2012-1726

28. Covic A, Mardare N, Gusbeth-Tatomir P, et al. Increased arterial stiffness in children on haemodialysis. Nephrol Dial Transplant. 2006;21(3):729-735. doi:10.1093/ndt/gfi196
Therapeutics and Clinical Risk Management

\section{Publish your work in this journal}

Therapeutics and Clinical Risk Management is an international, peerreviewed journal of clinical therapeutics and risk management, focusing on concise rapid reporting of clinical studies in all therapeutic areas, outcomes, safety, and programs for the effective, safe, and sustained use of medicines. This journal is indexed on PubMed Central, CAS,
EMBase, Scopus and the Elsevier Bibliographic databases. The manuscript management system is completely online and includes a very quick and fair peer-review system, which is all easy to use. Visit http://www.dovepress.com/testimonials.php to read real quotes from published authors. 